\section{Ultrasound-guided sclerotherapy using liquid sclerosant after preliminary saline flush}

\author{
Kenneth A. Myers, Amy May Clough \\ Victoria Vein Clinic, Melbourne, Australia
}

\section{Abstract}

The aim of this study is to evaluate the efficacy of liquid sclerosant with preliminary saline flush for ultrasound-guided sclerotherapy. Aethoxysklerol was used in varying concentrations ranging from $1.0 \%$ to $3.0 \%$ according to the volume required. Infusion of sclerosant was preceded by infusion of normal saline. Occlusion rates were determined by life-table analysis. Progressively lower concentrations were used as the study progressed. Life-table analysis showed significantly better results for tributaries compared to saphenous veins ( $92 \%$ vs $65 \%)$ and for dilute concentrations ( $\leq 1.5 \%)$ compared with stronger concentrations (>1.5\%) (88\% vs $66 \%)$. This observational study suggests that liquid sclerosant following a preliminary saline flush may give comparable results to those obtained with foam sclerotherapy. Confirmation would require a randomized controlled trial.

\section{Introduction}

Our unit has long advocated the use of foam for ultrasound-guided sclerotherapy. ${ }^{1}$ However, some four years ago we encountered increasing numbers of internet-wise patients who were aware of the findings by Morrison and Neuhardt that some $14-42 \%$ of patients studied with transcranial Doppler showed foam bubbles passing through the middle cerebral artery. $^{2}$ We emphasized to all patients that there was no evidence that foam sclerotherapy caused any form of brain damage except in a small fraction of the many patients being treated worldwide. ${ }^{3,4}$ However, we were unable to provide any evidence to reassure patients that the micro-bubbles seen in the middle cerebral artery in many patients treated with foam sclerotherapy was not a potential cause for diffuse brain damage. Since increasing numbers of patients then declined treatment with foam, we elected to return to using fluid sclerosant.

At about the same time, we became aware of in vitro studies by Parsi and colleagues that sodium tetradecyl sulphate was 30 times more effective and aethoxysklerol 160 times more effective in saline than in blood. ${ }^{5}$ The decision to find an alternative to foam and return to a liquid-based technique resulted in changing our in vivo technique to administer liquid aethoxysklerol in veins pre-flushed with normal saline.

The technique that then evolved is not new and was commonplace in many units including our own before the introduction of foam sclerotherapy.

\section{Materials and Methods}

This prospective observational study is of all patients treated by liquid ultrasound-guided sclerotherapy (UGS) by one phlebologist (KM) between April 2010 and August 2013. No patient was treated with foam sclerosant during this time. The treatment policy through this period was to offer endovenous laser ablation (EVLA) or mechanicochemical treatment (ClariVein ®; ClariVein Occlusion Catheter, Vascular Insights LLC, Madison, CT, USA) for all saphenous veins or major tributaries $>4 \mathrm{~mm}$ diameter. ${ }^{6}$ Liquid UGS was used as primary treatment for saphenous veins and major varicose tributaries $\leq 4 \mathrm{~mm}$ diameter. Associated smaller tributaries were not treated at the time of EVLA or ClariVein but left for two to three weeks to further reduce in size to be then treated by secondary UGS. Sclerotherapy under direct vision was used for small superficial varices, reticular veins and telangiectasias.

The series required 828 treatment sessions for 634 venous systems affecting 533 legs of 354 patients. Accordingly, multiple treatment sessions were frequently required (on average, 1.3 sessions per venous system). There were 81 male and 273 female patients and ages ranged from 17 to 83 (median 48) years. The clinical CEAP classifications were 429 C2-3, 48 C4, 2 C5 and 14 C6.

Treatment sessions were required for 275 venous systems not previously treated, 63 that had recurred after previous surgery, 262 treated at approximately two weeks after EVLA and 34 treated at approximately two weeks after ClariVein. The veins treated were 203 great saphenous veins, 41 anterior accessory saphenous veins, 47 small saphenous veins and 343 tributaries alone. Tributaries were treated for primary untreated disease for 88 sessions or recurrence after past surgery for 43 sessions (38\% for primary UGS), and after recent EVLA for 189 sessions and recent ClariVein for 23 sessions (62\% for secondary UGS).

All patients were studied before selecting treatment by standard techniques for duplex ultrasound as described by a International Union of Phlebology (UIP) consensus document. ${ }^{7}$ In particular, representative vein diameters were measured with the patient examined on a tilt table with the patient tilted head
Correspondence: Kenneth Myers, Victoria Vein Clinic, Suite 506, 100 Victoria Pde., East Melbourne, 3002, Victoria, Australia.

Fax: 61396634326.

E-mail: myers.kaba@gmail.com

Key words: sclerotherapy, saline flush, liquid sclerosant.

Received for publication: 20 September 2013.

Revision received: 9 January 2013.

Accepted for publication: 16 January 2013.

This work is licensed under a Creative Commons Attribution 3.0 License (by-nc 3.0).

(C) Copyright K.A. Myers and A.M. Clough, 2014

Licensee PAGEPress, Italy

Veins and Lymphatics 2014; 3:1933

doi:10.4081/vl.2014.1933

up at an angle of $45^{\circ}$. Reflux was defined as retrograde flow for $>0.5 \mathrm{~s}$ shown by spectral Doppler although the reflux time was appreciably greater in most patients. Studies were performed with the Philips IU 22 or GE Logic ultrasound machines.

\section{Preparation of sclerosant}

In Australia, the Therapeutic Goods Administration limit a daily dose of aethoxysklerol to no more than $2 \mathrm{mg} / \mathrm{kg}$ which is $4 \mathrm{ml}$ of $3 \%$ aethoxysklerol in an average size patient. The sclerosant was occasionally used undiluted but usually diluted with normal saline to provide larger volumes. If disease was extensive, it was common practice to treat each leg on separate days for bilateral disease. Progressively, lower concentrations were used and found to be at least equally effective so that it became frequent practice to make up 12 $\mathrm{mL}$ of $1 \%$ aethoxysklerol to be administered in $2 \mathrm{~mL}$ aliquots providing six injections. Similarly, a $1.2 \%$ solution allowed five injections, a 1.5\% solution allowed four injections, a $2 \%$ solution allowed three injections while a $3 \%$ solution allowed just two injections. The concentrations and volumes used for each session throughout the study are shown in Tables 1 and 2.

\section{Technique for injection}

The maximum volume at the selected concentration of aethoxysklerol is made up in a 10 mL syringe. A three-way tap is used with a $5 \mathrm{~mL}$ syringe containing normal saline in line with a $1 \frac{1}{2}$ inch 25 gauge needle and a $3 \mathrm{~mL}$ syringe containing a $2 \mathrm{~mL}$ aliquot of sclerosant to the side (Figure 1). The needle is passed into the vein confirmed by ultrasound and aspiration, the vein segment is then flushed with $5 \mathrm{~mL}$ of normal saline confirming that the needle is in the vein. In theory, $5 \mathrm{~mL}$ of saline should fill an approximate $10 \mathrm{~cm}$ length of a $4 \mathrm{~mm}$ diameter 
segment of vein. The three-way tap is then carefully turned with the free hand to allow the sclerosant to be infused. The vein is then vigorously massaged with the ultrasound probe for one to two minutes. It was found that this immediately helped to put the treated segment of vein into spasm. A disadvantage of the technique is the need to use two hands so that an assistant nurse or sonographer is required to hold the ultrasound transducer.

\section{Subsequent management}

After completing all injections, the treated limb is placed in a class I support garment (10-

Table 1. Number of sessions relating to concentration of sclerosant.

\begin{tabular}{cc}
$\begin{array}{c}\text { Concentration } \\
\text { sclerosant (\%) }\end{array}$ & $\begin{array}{c}\text { Number of } \\
\text { sessions }\end{array}$ \\
\hline 1.0 & 73 \\
1.2 & 49 \\
\hline 1.5 & 354 \\
2.0 & 319 \\
\hline 3.0 & 28 \\
\hline
\end{tabular}

Table 2. Number of sessions relating to volume of sclerosant.

\begin{tabular}{cc}
$\begin{array}{c}\text { Volume sclerosant } \\
(\mathrm{mL})\end{array}$ & $\begin{array}{c}\text { Number of } \\
\text { sessions }\end{array}$ \\
\hline 2 & 237 \\
3 & 105 \\
\hline 4 & 170 \\
\hline 5 & 41 \\
\hline 6 & 126 \\
\hline 8 & 76 \\
\hline 10 & 12 \\
\hline 12 & 27 \\
\hline
\end{tabular}

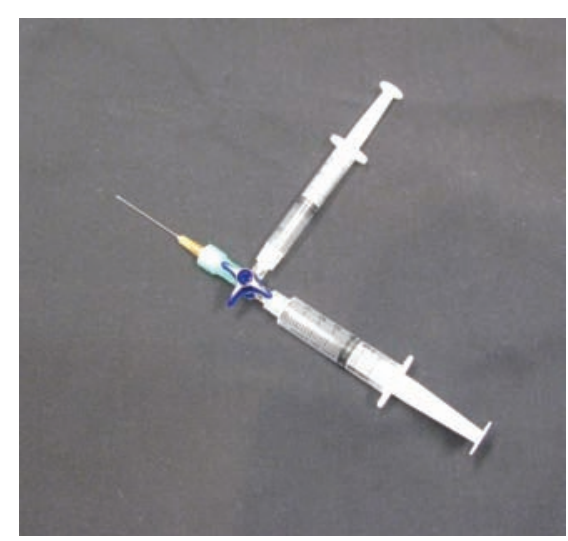

Figure 1. Syringes used for injection.
$19 \mathrm{mmHg}$ pressure) without bandaging or other forms of compression over the treated veins. The patient is then asked to walk for 10 to $15 \mathrm{~min}$. Compression is maintained through the first night after treatment and then during the day for as long as it is providing comfort to the patient. Daily walking is encouraged. All physical and sporting activities are allowed to be resumed after $24 \mathrm{~h}$.

\section{Follow-up}

Patients were reviewed clinically and with a duplex ultrasound scan to assess occlusion or persisting patency within a week after treatment. When the venous system was satisfactorily controlled, repeat ultrasound was performed at six weeks, six months then six

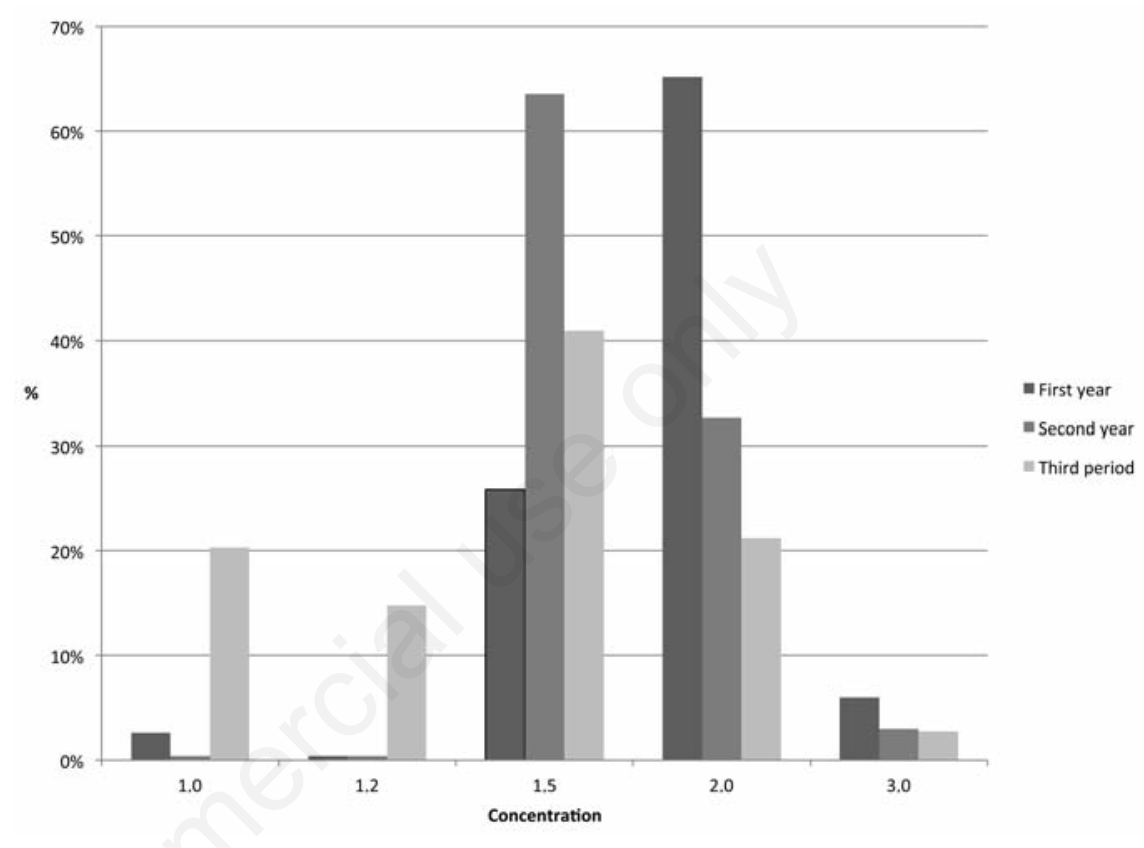

Figure 2. Concentrations according to veins treated.

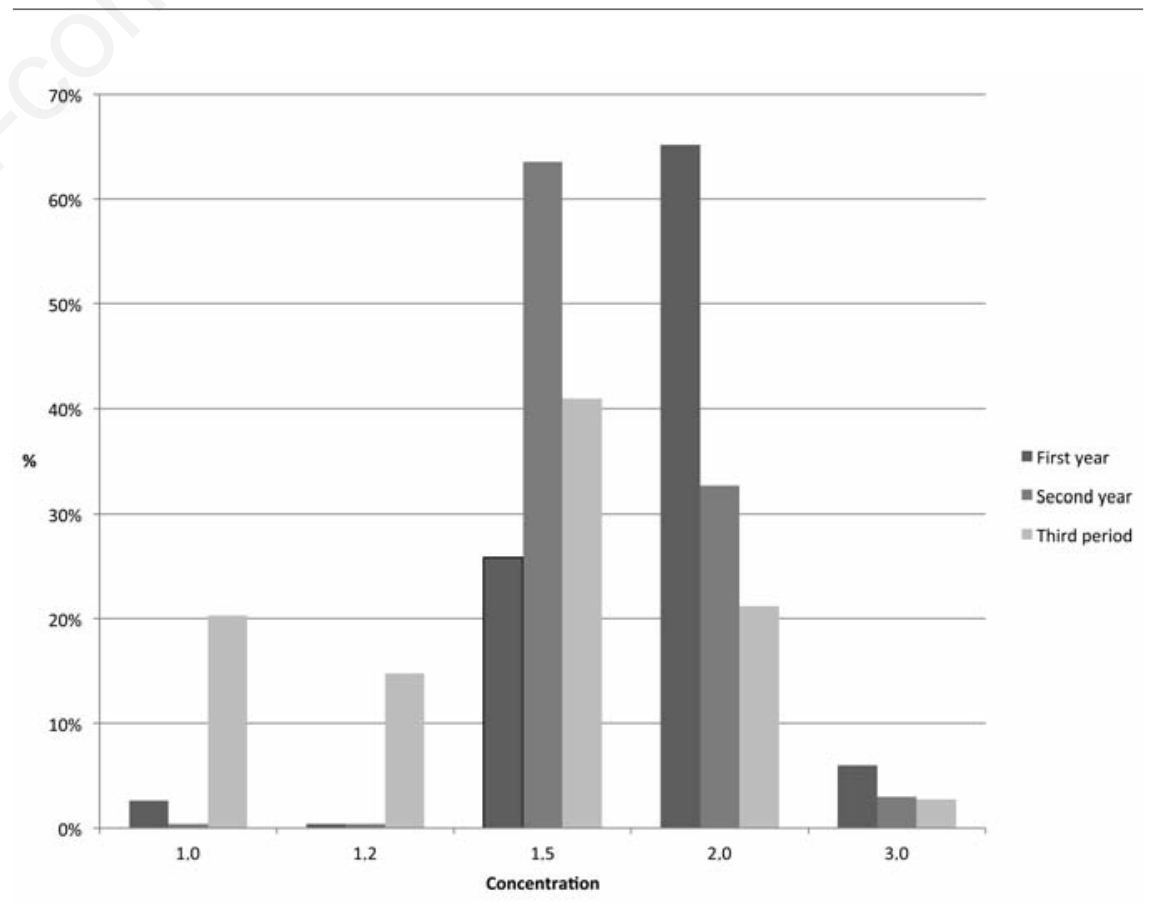

Figure 3. Concentrations according to time when treated. 
monthly until the treated veins could no longer be seen on ultrasound or until the present time.

\section{Analysis of outcome}

The only objective outcome measured was clinical and ultrasound occlusion of the veins. Actuarial life-table analysis for occlusion rates was performed with log-rank analysis for significance of difference between curves. To allow for censored data, results are shown to 12 months beyond which the standard error for cumulative failure rates exceeded $10 \%$.

\section{Results}

The most frequently used concentrations were $1.5 \%$ and $2 \%$. The $2 \%$ concentration was used more frequently for saphenous veins than for tributaries ( $46 \%$ vs $33 \%$ ) whereas the $1.5 \%$ concentration was used more frequently for tributaries that for saphenous veins (45\% vs 39\%) (Figure 2).

The numbers of sessions using different concentrations were arbitrarily divided into three time periods. The number of sessions using more dilute concentrations progressively increased through the study. During the first year from April 2010 to March 2011, 65\% of sessions used $2.0 \%$ concentration and $26 \%$ used $1.5 \%$ concentration. During the second year from April 2011 to March 2012, 33\% of sessions used $2.0 \%$ concentration and $65 \%$ used $1.5 \%$ concentration. During the third period from April 2012 to August 2013, 35\% of sessions used $1.2 \%$ or $1.0 \%$ concentration (Figure 3 ).

There were significantly better results for treatment for tributaries compared to all saphenous veins with $92 \%$ occlusion for tributaries and $65 \%$ occlusion for saphenous veins at 12 months (Figure 4). There was significant difference for occlusion rates according to the concentration of sclerosant with better results for more dilute concentrations compared to higher concentrations (Figure 5).

\section{Discussion}

Throughout the study, there were progressively increasing numbers of sessions using more dilute concentrations of sclerosant. This has the potential to confound results. It is difficult to compare the results for this study to those previously presented for foam sclerotherapy ${ }^{1}$ as it was policy then to treat larger diameter veins by UGS than at present. Indeed, it is difficult to compare results for studies from different groups because of variations in techniques to prepare foam. In vitro studies have shown marked variability of foam stability according to methods of preparation. ${ }^{8}$
However, occlusion rates at one year for all tributaries was $91 \%$ in the early study compared to $92 \%$ in the present study, while occlusion rates at one year for all great saphenous veins was $68 \%$ in the early study compared to $65 \%$ in the present study.

The finding of significantly better results for dilute compared to more concentrated solutions in the present study is in part due to a moderately higher proportion of saphenous veins in the latter group but is in large part unexplained, and further work will be needed to explain this apparent paradox. It is in keeping with findings in the earlier study using foam where multivariate analysis showed that failures occurred in $14 \%$ of veins treated by $1.5 \%$ concentration compared to $34 \%$ treated by $3.0 \%$ concentration independent of the veins treated.

It must be again emphasized that there have

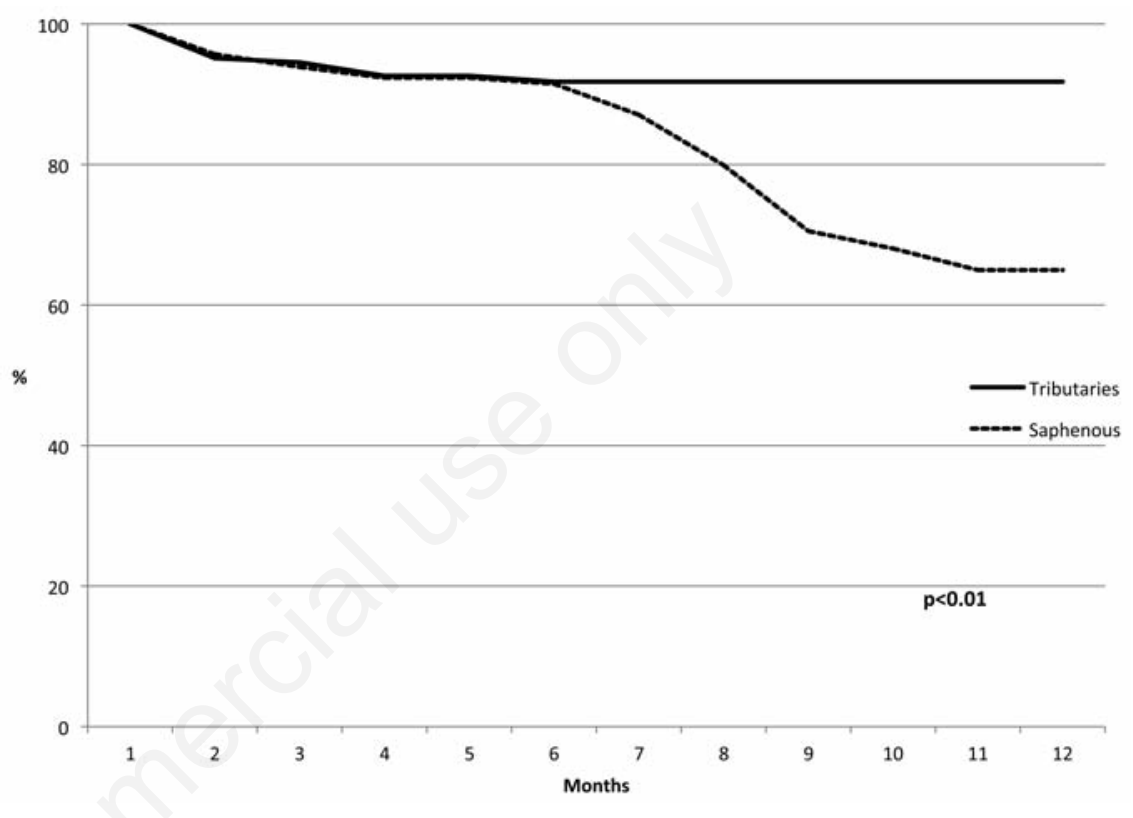

Figure 4. Primary patency rates saphenous versus tributaries.

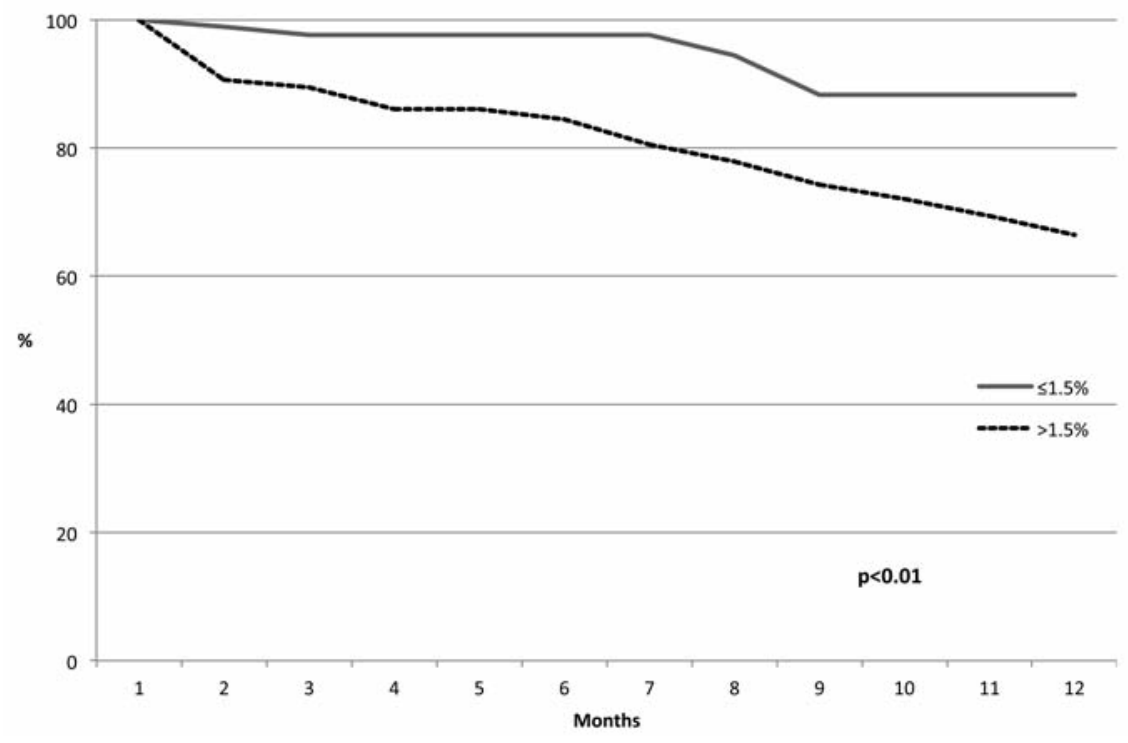

Figure 5. Primary patency rates according to concentration. 
techniques for foam sclerotherapy carry any risk of causing brain damage.

extremely few patients reported as fering from stroke among the many millions of patients treated by foam sclerotherapy. However, this failed to reassure our patients that such treatment could be associated with diffuse brain damage from micro-bubbles. This will require studies using sophisticated magnetic resonance imaging techniques before and after treatment. It is also possible that other techniques to treat venous disease such as endothermal ablation or the combination of mechanical ablation and sclerosants are associated with production of bubbles or particulate matter that pass to the cerebral arteries. Furthermore, adverse effects may result from other mechanisms, particularly release of chemicals from injured endothelial cells.

Since this is a preliminary observational study, the efficacy of the present treatment would need to be evaluated by a prospective randomized controlled trial. This would be worthwhile if it is considered that current

\section{References}

1. Myers KA, Jolley D, Clough A, Kirwan J. Outcome of ultrasound-guided sclerotherapy for varicose veins: medium-term results assessed by ultrasound surveillance. Eur J Vasc Endovasc Surg 2007;33: $116-21$.

2. Morrison N, Neuhardt DL. Foam sclerotherapy: cardiac and cerebral monitoring. Phlebology 2009;24:252-9.

3. Rathbun S, Norris A, Stoner J. Efficacy and safety of endovenous foam sclerotherapy: meta-analysis for treatment of venous disorders. Phlebology 2012;27:105-17.

4. Guex JJ. Complications and side-effects of foam sclerotherapy. Phlebology 2009;24: 270-4.

5. Parsi K, Exner T, Connor DE, et al. The lytic effects of detergent sclerosants on erythrocytes, platelets, endothelial cells and microparticles are attenuated by albumin and other plasma components in vitro. Eur J Vasc Endovasc Surg 2008;36:216-23.

6. Myers KA, Clough A, Tilli H. Endovenous laser ablation for major varicose tributaries. Phlebology 2013;28:180-3.

7. Coleridge-Smith P, Labropoulos N, Partsch $\mathrm{H}$, et al. Duplex ultrasound investigation of the veins in chronic venous disease of the lower limbs-UIP consensus document. Part I. Basic principles. Eur J Vasc Endovasc Surg. 2006;31:83-92.

8. Cameron E, Chen T, Connor DE, et al. Sclerosant foam structure and stability is strongly influenced by liquid air fraction. Eur J Vasc Endovasc Surg 2013;46:488-94. 